\title{
B1,3-GLUCAN ANTICANCER EFFICACIES AND SYNERGIES: A REVIEW
}

\author{
${ }^{1}$ Story Jenny, ${ }^{2}$ Vetvicka Vaclav and ${ }^{1}$ Angove Michael \\ ${ }^{1}$ School of Pharmacy, Faculty of Science, Technology and Engineering, La Trobe University, Australia \\ ${ }^{2}$ Department of Pathology, University of Louisville, Louisville, KY 40202, USA
}

Received 2014-07-22; Revised 2014-08-06; Accepted 2014-08-12

\begin{abstract}
$\beta 1,3$-glucans from fungi, cereals, seaweeds and bacteria have been shown to possess favourable biological and anti-carcinogenic activities including upregulation of phagocytosis, cytokine production enhancement, superoxide and nitrite production; antibody secretion and stimulation of signalling pathways associated with proto-oncogene expression. However, human dietary supplements containing $\beta 1,3$-glucans vary in efficacy due to glucan source, the lifecycle stage of the source at extraction, extraction methods, purity, concentration and combination with other immunomodulators. A review of efficacy of some commercially available $\beta 1,3$-glucan products is presented. Three apparently efficacious products in which $\beta 1,3$-glucan was the only immunomodulator were identified: Glucan \#300®, Maitake Gold 404® (diluted Yukiguni Maitake MD Fraction ${ }^{\circledR}$ ) and Betamune ${ }^{\circledR}$. A trial of Maitake Gold 404® produced evidence of standardisation problems. It is recommended that Yukiguni Maitake MD Fraction® (a more standardised alternative), Glucan \#300® and Betamune ${ }^{\circledR}$ be comparatively trialled at optimal doses across immunological measures and tumor reduction. $\beta 1,3$-glucans have been shown to be synergistic with conventional cancer therapies and monoclonal antibodies, as well as immunomodulators including vitamin $\mathrm{C}$, transresveratrol, humic acids and Ashwagandha (Withania somnifera). Trialled commercially available products containing immunomodulator combinations have been shown to be inefficacious, apart from RVB300®, a $\beta 1,3$-glucan/transresveratrol/vitamin C combination. The efficacies of various combinations of $\beta 1,3$-glucans with other immunomodulators and the details of specific $\beta 1,3$-glucan/monoclonal antibody synergies in treating particular cancer cell lines, require systematic elucidation.
\end{abstract}

Keywords: Cancer, Chemotherapy, Glucan, Immunomodulators, Monoclonal Antibodies

\section{INTRODUCTION}

The estimated number of Americans to receive a new cancer diagnosis in 2014 is 1,665,540, with almost 1,600 deaths per day predicted (ACS, 2014). Cancer is the cause of one in every four American deaths (ACS, 2014). Marked morbidities are associated with both cancer and chemotherapy (Nanba, 1997). The identification of novel pharmaceuticals containing effective treatment agents, is essential for evidence based pharmacy practice.Possible agents include $\beta$ glucans: Complex, structurally diverse $\beta$-glucose homopolymers isolated from the cell walls of grains, fungi, seaweeds and bacteria (Jamois et al., 2005; Novak and Vetvicka, 2008; 2009; Vetvicka and Vetvickova, 2010; Vetvicka et al., 2002; Vetvicka et al., 2007a; Vetvicka and Yvin, 2004). Nomenclature designates glycosidic bond positions, position $\beta(1,3)$ with side chains bound by 1,6 glycosidic linkages predominant in studies, due to their allegedly superior immunological and anti-carcinogenic activity (Novak and Vetvicka, 2009). Degree of branching, detailed structure and solubility and hence biological activity of $\beta 1,3$-glucans differ according to source and isolation method (CMEC,

Corresponding Author: Jennifer Story, School of Pharmacy, Faculty of Science, Technology and Engineering, La Trobe University, Australia Tel: +61 428397219 Fax: +61 354447976 
2006; Liu et al., 2009; Lowman et al., 2003; Novak and Vetvicka, 2008; 2009; Tada et al., 2007; 2009; Vetvicka et al., 2002; Zhang et al., 2007).

\subsection{B1,3-Glucan Anti-Cancer Immunological Effects and Applications}

Tumoricidal properties of polysaccharides have been researched since the 1940s (Novak and Vetvicka, 2008; Vetvicka and Vetvickova, 2011a) and by 2010 the active immunomodulatory properties of $\beta 1,3$-glucans, including anti-bacterial and anti-tumoractivity, had been demonstrated in over 6,000 papers and across all tested species (Novak and Vetvicka, 2009; Vetvicka and Vetvickova, 2010; Vetvicka et al., 2007a). Anti-tumor activities have been shown in cancer models including colorectal (Vetvicka et al., 2002), lung (Vetvicka et al., 2007a), mammary (Vetvicka and Yvin, 2004) and gastric cancer (Shomori et al., 2009). Japanese studies have repeatedly asserted the anti-cancer effects of Maitake $\beta$ glucan MD fraction, from the mushroom Grifola frondosa, also termed "D fraction" (Hishida et al., 1988; Inoue et al., 2002; Kodama et al., 2002a; 2002b; Masuda et al., 2008; Nanba, 1997; Nanba et al., 1987), notably in human subjects with breast, liver or lung cancers (Kodama et al., 2002a; Nanba, 1997).

Unlike other natural immunomodulators, purified $\beta 1,3$ glucans retain their bioactivity for a long time after administration, facilitating characterisation of their anticancer mechanisms of action at molecular and cellular levels (Hong et al., 2004; Kodama et al., 2002b; Vetvicka and Vetvickova, 2007a). B1,3-Glucans are treated as pathogens by pattern recognition receptors on macrophages, neutrophils, monocytes and Natural Killer cells (NK cells) (Hong et al., 2004; Kodama et al., 2002a; Liu et al., 2009; Novak and Vetvicka, 2009; Vetvicka and Vetvickova, 2005). $\beta 1,3$-Glucans from different sources possess differential receptor affinities (Liu et al., 2009), most importantly for Complement Receptor 3 (CR3) on NK cells, macrophages and neutrophils and Dectin-1 receptor on macrophages (Hong et al., 2004; Li et al., 2006; Liu et al., 2009; Masuda et al., 2012; Novak and Vetvicka, 2008; 2009; Vetvicka and Vetvickova, 2005; Vetvicka and Vetvickova, 2011a; Vetvicka and Yvin, 2004; Vetvicka et al., 2007a; Vetvicka et al., 2013; Wu et al., 2006).

After $\beta 1,3$-glucan-CR3 binding, granulocytes specifically attack iC3b-opsonized tumor cells and pathogens (Li et al., 2006). Targeting leads to enhanced phagocytosis by macrophages, monocytes and neutrophils (Vetvicka and Vetvickova, 2007b; Vetvicka and Yvin, 2004; Vetvicka et al., 2007a; Wu et al., 2006), followed by superoxide and nitrite production (Nanba, 1997; Novak and Vetvicka, 2009; Vetvicka and Vetvickova, 2010), then pro-and anti-inflammatory cytokine secretion enhancement (Deng et al., 2009; Jamois et al., 2005; Kodama et al., 2002a; Nanba, 1997; Novak and Vetvicka, 2008; 2009; Shomori et al., 2009; Vetvicka and Vetvickova, 2005; Vetvicka and Vetvickova, 2007b; Vetvicka and Yvin, 2004; Vetvicka et al., 2002; Wu et al., 2006; Xu et al., 2010), tumor regression and enhanced host survival (Hong et al., 2004; Kodama et al., 2002a; Vetvicka and Yvin, 2004; Zhang et al., 2007). The relevant cytokines can function either as growth factors or cancer growth inhibitors and include interleukins such as IL-1, IL-2, IL-6, IL-10, IL12 , IFN- $\gamma$ and TNF- $\alpha$ (Deng et al., 2009; Jamois et al., 2005; Kodama et al., 2002a; 2002b; Nanba, 1997; Tada et al., 2007; Vetvicka and Vetvickova, 2005; Vetvicka and Vetvickova, 2007a; Vetvicka and Yvin, 2004; Wu et al., 2006; Xu et al., 2010). Also via CR3 binding, $\beta 1,3$-glucan administration inhibits the adhesion molecules favouring metastasis (Masuda et al., 2008; Novak and Vetvicka, 2009).

Additional interrelated immunological events following receptor binding include NK cell activity (Hamano et al., 1999; Kodama et al., 2002a; 2002b; Nanba, 1997; Vetvicka and Vetvickova, 2005; Vetvicka and Yvin, 2004; Wu et al., 2006); granulocyte (Ito et al., 2009; Lin et al., 2007) and $\mathrm{CD}^{+}, \mathrm{CD}^{+} \mathrm{T}$ and B lymphocyte migration (Vetvicka and Vetvickova, 2005; Vetvicka and Vetvickova, 2007b; Vetvicka et al., 2002; Xu et al., 2010); and increase of antibody production (Vetvicka and Vetvickova, 2007b; Vetvicka and Vetvickova, 2010; Vetvicka and Vetvickova, 2012a; Vetvicka et al., 2007a; Xu et al., 2010). B1,3-glucan stimulates the Syk-phosphatidylinoitol 3-kinase and phosphorylated extracellular signal-regulated kinase signalling pathways (Li et al., 2006; Masuda et al., 2012), affecting expression of genes involved in cancer cell proliferation, invasion and apoptosis (Novak and Vetvicka, 2009; Vetvicka et al., 2007b; Vetvicka et al., 2008; Xu et al., 2010; Zhang et al., 2007). The question of the genetic landscape underlying cancers is complex: The simultaneous over-or under-expression of specific combinations of genes is closely associated with carcinogenesis. Further, relevant genes may possess a range of isoforms which differentially promote or suppress cell growth and which are differentially expressed by normal or cancerous cells (Novak and Vetvicka, 2009; Otake et al., 2007). 


\subsection{Potential Sources of Variability in $\beta 1,3-$ Glucan Product Efficacy}

The concentration of effective $\beta$-glucan in a product and the dose bear a strong relationship to immunological effects, as evidenced by (Deng et al., 2009), who measured the human oral dose response curves for Maitake Gold 404® (containing Yukiguni Maitake MD Fraction $\left.{ }^{\circledR}\right)$ across a wide range of immunological parameters. Optimal dose for many parameters was around 5-7 mg/kg/day, with highest dose causing reductions in some immunological reactions. The effective dose of $\beta$-glucan within a product is affected by source, growth stage of the source, purity and extraction method.

Unfortunately, a significant part of research has been conducted with only partially purified $\beta 1,3$-glucans, making assessment of efficacies of specific glucans problematic (Vetvicka and Vetvickova, 2007a; Xu et al., 2010). Individual fungal species incorporated in products may contain multiple ingredients which can contribute to immunological and biological activity (Vetvicka and Vetvickova, 2007a), including many types of $\beta$-glucans: Numerous different $\beta 1,3$-glucans have been identified in Maitake mushrooms and Saccharomyces cerevesiae or Candida albicans yeasts alone (Cui et al., 2007; Ito et al., 2009; Lowman et al., 2003; Ohno et al., 1986; Tada et al., 2009; Vetvicka and Vetvickova, 2005; Zhang et al., 2007). There is significant growth condition-related variation in $\beta 1,3$-glucan branching and molecular weight within individual sources and hence variable potentially anti-tumor properties (Jamois et al., 2005; Lowman et al., 2003; Novak and Vetvicka, 2009).

Further heterogeneity of immunological efficacy between commercially available $\beta 1,3$-glucan products relates to isolation methods (CMEC, 2006; Vetvicka and Vetvickova, 2005). For example, two different methods of preparation of the $\beta$-glucan Maitake MD fraction produce $\beta$-glucans which are protein bound, or otherwise: Maitake Gold 404® and Grifron Pro Maitake D Fraction® respectively (Vetvicka and Vetvickova, 2005).

The increased heat and alkalic solvents commonly used in extraction may destroy triple helical structures underlying some mechanisms of action in highly branched $\beta 1,3$-glucans (Novak and Vetvicka, 2008; 2009; Ohno et al., 1986; Vetvicka and Vetvickova, 2010; Zhang et al., 2007), such as Maitake MD fraction. Opinions vary as to whether, following hydrolytic removal of Maitake MD fraction side branches, the fraction lacks immunostimulatory activity (Lin et al., 2009; Miyake et al., 2005). Therefore, hot water extraction is recommended above the less costly alcohol extraction, which removes side branches and may destroy some efficacy (Zhang et al., 2007).

Poorly isolated $\beta 1,3$-glucans have comparatively small biological and immunological effects and in some cases may be even toxic (CMEC, 2006; Jamois et al., 2005; Novak and Vetvicka, 2008; Vetvicka and Vetvickova, 2010). However, soluble glucans can be further fractionated (precipitated with various concentrations of ethanol) so as to obtain a product with specific chemical composition and enhanced purity and efficacies (Nanba, 1997; Wu et al., 2006; Zhang et al., 2007). Yukiguni MD Fraction ${ }^{\circledR}$ from Maitake mushroomsis hot water extracted and fractionated (Kodama et al., 2002a; Nanba and Kubo, 1997).

The burgeoning of $\beta 1,3$-glucan products commercially available as human dietary supplements (in 2014 over 400 on the U.S. market alone) raises the importance of identification of those with superior anticarcinogenic properties. Proprietary extracts from yeast alone vary in manufacturer, solubility and purity (Vetvicka and Vetvickova, 2010; Vetvicka et al., 2008). With the notable exception of the work of Vetvicka's group, direct comparisons of immunological and biological actions of $\beta 1,3$-glucan products are almost non-existent (Vetvicka and Vetvickova, 2005; Vetvicka and Vetvickova, 2007a; 2007b; 2009; 2010; Vetvicka et al., 2008). From the perspective of cancer patients, evidencebased use of such products is critical.

Furthermore, $\beta 1,3$-glucan products for human consumption containing additions of any number of other alleged immunomodulators, including Astralagus, Echinacea, Aloe vera or Hydrastis Canadensis (Goldenseal) are increasingly available (Vetvicka and Vetvickova, 2012c). The immunological effects and toxicities of such combinations are generally unknown. It is crucial to patient interests that combinations of commercially available $\beta 1,3$-glucans with anti-cancer medications or other immunomodulators be evidence based.

\section{COMPARATIVE STUDIES OF POTENTIALLY ANTI-CANCER IMMUNOMODULATION BY $\beta 1,3-$ GLUCAN PRODUCTS}

In 2005 Vetvicka and Vetvickova compared the immunostimulating properties of lentinan and two different preparations of Maitake D or MD fraction: Maitake Gold 404® containing diluted patented MD fraction from Yukiguni biofarm, a $\beta 1,3$-glucan-protein complex (Nanba and Kubo, 1997) and Grifron-Pro 
Maitake D Fraction ${ }^{\circledR}$, a $\beta 1,3$-glucan: Agents 1, 2 and 3 on Table 1 (Vetvicka and Vetvickova, 2005). Following oral administration to mice, all three preparations stimulated phagocytosis to the same extent. However, Maitake Gold 404® was the only product effective at low doses. It produced large between-sample phagocytosis stimulation variation specifically of neutrophils, suggesting a lack of standardisation of effective MD fraction concentration.

Unlike lentinan, both Maitake extracts stimulated production of $\mathrm{CD}^{+} \mathrm{T}$ cells. Lentinan took longer to stimulate IL- $1 \beta$ and only Maitake Gold 404® stimulated TNF- $\alpha 60$ min after administration. Lentinan and Grifron-Pro Maitake D Fraction ${ }^{\circledR}$ were most effective at stimulating IL-2 production, whereas Maitake Gold $404 ®$ scored slightly under control, an anomalous result again suggesting effective concentration issues. It is to be noted that, in general, almost all $\beta 1,3$-glucans stimulate IL-2 production (Deng et al., 2009; Nanba, 1997; Vetvicka and Vetvickova, 2007a; Vetvicka and Vetvickova, 2007b; Vetvicka and Vetvickova, 2010).

All glucans enhanced NK activity, but Maitake Gold 404® had the strongest effect. The researchers concluded that $\beta 1,3$-glucans from Maitake possess similar types of immunomodulatory properties to those from yeasts and that in some ways they are more immunologically active.
Vetvicka and Vetvickova later conducted a review of the magnitude of some immunological effects of a range of $\beta 1,3$-glucan human dietary supplements (Table 2), in which $\beta 1,3$-glucan was the only potentially immunomodulatory agent (Table 1 agents 2,4-9), (Vetvicka and Vetvickova, 2007b). In this study, Now Beta ${ }^{\circledR}$, Immutol®, Immune Builder ${ }^{\circledR}$, Glucan \#300® and Maitake Gold 404® stimulated the greatest rates of phagocytosis.

The three agents stimulating the greatest migration of $\mathrm{CD}^{+}$and $\mathrm{CD}^{+} \mathrm{T}$ lymphocytes were Now Beta ${ }^{\circ}$, Glucan \#300® and Maitake Gold 404®. Glucan \#300® and Maitake Gold404® stimulated the highest IL-2 production. Glucagel $T \AA$ and Glucan $\# 300 \AA$ stimulated the highest antibody response. Although it was possible for an agent to score highly on one measure of immunomodulation and negligibly on others (for example, Glucagel $\mathrm{T} @$ stimulated well antibody formation, but on no other activity), clearly the most active agents across immunological measures were Glucan \#300®, Now Beta ${ }^{\circledR}$ and Maitake Gold 404®. Senseiro®'s apparently low immunological activity was contrary to a study asserting the anti-tumour properties of Agaricus blazei fractions (Kawagishi et al., 1989). It might be possible that A. blazei contains components lacking in Senseiro ${ }^{\circledR}$ and responsible for its anti-tumor activity.

Table 1. A partial list of $\beta 1,3$-glucan products commercially available as human food supplements

1,3-glucan product
1) Lentinan
2) Maitake Gold $404 \AA$
3) Grifron-Pro Maitake®
4) Glucan \# $300 \AA$
5) Now Beta $($ also called “now Glucan” ()
6) Glucagel T®
7) Immutol ${ }^{\circledR}$
8) Immune Builder $\AA$

9) Senseiro®

10) Krestin ("PSK")

11) ImmunoFibre

12) Wellmune ${ }^{\circledR}$

13) Betamune ${ }^{\circledR}$

14) Epicor ${ }^{\circledR}$

15) Aktival ${ }^{\circledR}$

16) Sweet Beta Glucan ${ }^{\circledR}$

17) Beta Right $101{ }^{\circledR}$

18) NSC®

19) Better Immunity®

20) Macroforce ${ }^{\circledR}$

21) Solgar®

22) Swanson ${ }^{\circledR}$
Source

Fruit body of Lentinula edodes (Shiitake) mushroom

Grifola frondosa (Maitake) mushroom ${ }^{\text {a) }}$

Grifola frondosa (Maitake) mushroom ${ }^{\text {a) }}$

Yeast: Highly purified Whole Glucan Particle (WGP)

Yeast, mushrooms

Barley

Yeast

5 mushrooms: Agaricus blazei, Coriolus versicolor,

Ganoderma lucidum (Reishi), Lentinula edodes,

Grifola frondosa

Agaricus blazei Murill Kyowa mushroom

Coriolus versicolor (Trametes versicolor) mushroom

Barley

Saccharomyces cerevisiae WGP

Saccharomyces cerevisiae

Saccharomyces cerevisiae

Saccharomyces cerevisiae

Saccharomyces cerevisiae

Saccharomyces cerevisiae

Saccharomyces cerevisiae

Saccharomyces cerevisiae

Sacharomyces cerevisiae

Saccharomyces cerevisiae

Saccharomyces cerevisiae 
Story Jenny et al. / American Journal of Immunology 10 (3): 131-143, 2014

Table 2. 31,3 -glucan products found to be most efficacious immunomodulators in comparative studies

\begin{tabular}{l}
\hline Most effective product \\
\hline Maitake Gold404® \\
Now Beta® \\
Immutol ${ }^{\circledR}$ \\
Immune Builder® \\
Glucan \#300® \\
Maitake Gold 404® \\
Now Beta $®$ \\
Glucan \#300® \\
Maitake Gold 404® \\
Glucan \#300® \\
Maitake Gold 404® \\
Glucagel T® \\
Glucan \#300® \\
Glucan \#300® \\
Now Beta® \\
Maitake Gold 404® \\
Glucan \#300® \\
Glucan \#300® \\
Krestin \\
Krestin \\
Glucan \#300® \\
Betamune®
\end{tabular}

Glucan \#300®

\section{Glucan \#300®}

Glucan \#300®

Wellmune®

Maitake Gold 404®,

Krestin,

Glucagel T®

Glucan \#300®

Wellmune ${ }^{\circledR}$

Maitake Gold 404®,

Krestin

Beta Right ${ }^{\circledR}$

Glucan \#300®

Wellmune ${ }^{\circledR}$

Beta Right ${ }^{\circledR}$

Glucagel T®

Immunological measure

Phagocytosis

CD4+ and CD8+ T lymphocyte migration

IL-2 production

Antibody formation

Most active across measures

Greatest phagocytosis

Longest lasting phagocytosis

IL-2 production

IL-2 production, first 2 days

Most active at lower doses

Phagocytosis in widest effector cell range

Generally strongest cytokine response

Greatest tumor weight reduction

Increased expression of the widest range of proto-oncogenes

Recovery of phagocytosis

Cytokine production.

$\mathrm{T}$ and $\mathrm{B}$ cell proliferation and NK cell activity after mercury instigated immunosuppression.

Phagocytosis

Superoxide and nitrite production

IL-2 production

Antibody production
Study

Vetvicka and Vetvickova (2005)

Vetvicka and Vetvickova (2007b)

Vetvicka and Vetvickova (2007b)

Vetvicka and Vetvickova (2007b)

Vetvicka and Vetvickova (2007b)

Vetvicka and Vetvickova (2007b)

Vetvicka and Vetvickova (2007a)

Vetvicka and Vetvickova (2007a)

Vetvicka and Vetvickova (2007a)

Vetvicka and Vetvickova (2007a)

Vetvicka et al. (2008)

Vetvicka and Vetvickova (2009)

Vetvicka and Vetvickova (2010)

Vetvicka and Vetvickova (2010)

Vetvicka and Vetvickova (2010)

Vetvicka and Vetvickova (2010)
In an additional study (Vetvicka and Vetvickova, 2007a), the same group examined murine cholesterol and blood glucose level changes, as well as immunological changes (phagocytosis by peripheral leukocytes, IL-2 production) following administration of Glucan \#300®, Now Beta®, Krestin and ImmunoFiber ${ }^{\circledR}$ (agents 4, 5, 10 and 11 respectively, Table 1). Glucan \#300® stimulated greatest phagocytosis and, along with Krestin, longest lasting increases in that measure. IL-2 production was most lastingly stimulated by Krestin, although the highest level of stimulation in the first two days after $\beta 1,3$ glucan administration was by Glucan \#300®. Glucan $\# 300 \circledR$ was again the most active sample.

Vetvicka et al. (2008) examined changes in the release of seven cytokines and levels of phagocytosis in 
a murine and in vitro model following administration of different doses of $\beta 1,3$-glucans WGP, Lentinan and Betamune ${ }^{\circledR}$ (agents 1, 12 and 13, Table 1). In vivo tumor inhibition and breast cancer related gene expression were also examined. WGP and Lentinan were only active at the highest dose. Betamune ${ }^{\circledR}$ was more active at lower doses than the otheragents, stimulated phagocytosis in a wider effector cell range, in general stimulated the strongest cytokine response and resulted in the greatest tumor weight reduction. It also stimulated increased expression of the widest range of protooncogenes, potentially associated with upregulation of the adhesion molecules and angiogenesis contributing to tumor metastasis and growth, a finding similar to that in other studies on glucans (Vetvicka et al., 2007b). This result should be considered in light of the complexities of the genetic landscapes underlying cancers (Novak and Vetvicka, 2009; Otake et al., 2007).

Another study compared the $\beta 1,3$-glucan-mediated recovery of phagocytosis, production of three cytokines, $\mathrm{T}$ and $\mathrm{B}$ cell proliferation and NK activity after mercuryinstigated immunosuppression (Vetvicka and Vetvickova, 2009). Following administration of $\beta 1,3$-glucans $4-6$ and 13 (Table 1), Glucan \#300® was the only agent active on all measures and was consistently most active.

An additional comparative study evaluated sixteen commercially available $\beta 1,3$-glucan products in terms of immunological activity (Table 1, agents 2, 4-7, 10, 12 and 14-22) and tested phagocytosis, antibody formation, phagocyte superoxide and nitrite production and cytokine production (Vetvicka and Vetvickova, 2010). Only Glucan \#300® stimulated phagocytosis across dose ranges. The glucans most active in stimulating superoxide and nitrite production were Glucan \#300®, Wellmune®, Maitake Gold 404®, Krestin and Glucagel T®. Glucans stimulating the highest IL-2 production included Glucan \#300®, Wellmune®, Maitake Gold 404®, Krestin and Beta Right®. The highest antibody production was produced by Glucan \#300®, Wellmune ${ }^{\circledR}$, Beta Right ${ }^{\circledR}$ and Glucagel T®.

As in previous comparative study, many of the glucans tested (e.g., Swanson $®)$, showed little immunological activity and some agents produced a high level of activity on some measures only. Glucan \#300® showed the greatest activity across all measures (Table 2). The authors have repeatedly hypothesised that this was partly because Glucan \#300® had a high degree of purity compared to other agents: For example, Glucan \#300® is $85 \%$ pure and Swanson® 70\% (Vetvicka and Vetvickova, 2010).

\section{3. $\beta 1,3-G L U C A N$ SYNERGIES WITH ANTI-CANCER MEDICATIONS AND IMMUNOMODULATORS}

Clinical and experimental evidence has clearly established the anti-cancer synergy of a range of $\beta 1,3-$ glucans with conventional cancer therapies including cyclophosphamide (Tada et al., 2007; Vetvicka et al., 2007a), interferon- $\alpha$ (Louie et al., 2010), mitomycin C (Nanba, 1997), dendritic cell-based immunotherapy (Masuda et al., 2010) and radiotherapy (Gu et al., 2005). Kodama et al. (2002a) published a non-randomised clinical trial in which Yukiguni Maitake MD Fraction ${ }^{\circledR}$ plus whole Maitake powder, taken with various chemotherapies, enhanced immunocompetent activities by a factor of 1.2-1.4 compared with chemotherapy alone.A previous limited, non-controlled study published by Nanba found 12-28\% improved efficacy of the whole Maitake MD fractionand chemotherapy above that of chemotherapy (Nanba, 1997). Both studies found tumor regression or complete remission in some patients. However, the MD fraction doses administered were significantly under the optimal ranges determined by (Deng et al., 2009) and the greater efficacy achieved with addition of whole Maitakepowder may have been the result of additional MD fraction related $\beta$-glucans in the powder rather than any true synergy of fraction and powder. A commercially available MD Fractionwhole Maitake powder product derived from Yukiguni Maitake and combined approximately in the ratio used in the Nanba study is Maitake QOL Extract ${ }^{\circledR}$ (Muir, 2012; Nanba, 1997).

Diverse $\beta 1,3$-glucans ameliorate the neutropenia, bone marrow hematopoietic cell depression and secondary infections prevalent with conventional treatments including methotrexate (Sener et al., 2006), cyclophosphamide (Vetvicka et al., 2007a), 5-fluorouracil (Vetvicka et al., 2007a), doxorubicin (Lin et al., 2004), paclitaxel (Lin et al., 2010), cisplatin (Masuda et al., 2009), mitomycin C (Nanba, 1997) and X-ray and radiation therapy ( $\mathrm{Gu}$ et al., 2005). The evidence for Maitake MD fraction in this regard is notable in terms of numbers of studies (Kodama et al., 2002a; Lin et al., 2004; 2007; 2009; 2010; Louie et al., 2010; Masuda et al., 2009; Nanba, 1997). Human subjects administered MD fraction also experience reduced chemotherapy side effects, including pain, lost appetite, vomiting, nausea and hair loss (Nanba, 1997). 


\subsection{Monoclonal Antibodies}

Combination of anti-tumor monoclonal antibodies (mAbs) with standard chemotherapy increases mAb efficacy, but adverse effect incidence and severity increase to an extent that limits utilisation (Liu et al., 2009). Enhanced effectiveness of immune responses to a range of cancers (including breast, lung and lymphoma), tumor shrinkage and prolongation of survival have repeatedly been demonstrated in murine and human xenograft models with co-administration of complement activating mAbs to tumor antigens, plus $\beta 1,3$-glucans. The latterincludethe commercially available Glucan \#300 and WGP: Agents 4 and 12 on Table 1, as well as a related yeast $\beta 1,3$-glucan, PGG and a barley derived $\beta 1,3$-glucan (Hong et al., 2004; Liu et al., 2009; Modak et al., 2013; Salvador et al., 2008; Yan et al., 2005).

PGG has the advantage of being the only tested $\beta 1,3$ glucan product which does not amplify inflammatory cytokine responses, a desirable attribute in mAb therapy (Liu et al., 2009). Unfortunately it is only available to patients via trials. Bevacizumab, an anti-vascular endothelial growth factor (VEGF) mAb, inhibits tumor microvasculature and triggers $\mathrm{iC} 3 \mathrm{~b}$ deposition on surface vasculature of a human ovarian carcinoma cell line, thus making such cells a target for PGG $\beta$-glucan primed neutrophil CR-3 related toxicity (Liu et al., 2009; Salvador et al., 2008). In one study, combination therapy prevented tumors from growing and led to long-term survival in $86 \%$ of murine subjects with tumors (Salvador et al., 2008). However, the combination provided no benefit above mAb alone in Colo38 tumors, since they do not express membrane bound VEGF (Salvador et al., 2008). Therefore the identification of cancer types possessing membrane-bound VEGF is crucial for the selection of patients to undergo combination PGG $\beta$-glucan bevacizumab therapy.

Similarly, cetuximab, a humanised anti-Epidermal Growth Factor Receptor (EGFR) mAb, when combined with PGG reduced tumor burden and greatly increased the rate of murine long-term survival (Liu et al., 2009). EGFR is highly expressed in very aggressive colorectal, lung, breast, pancreatic and gastrointestinal tumors and melanoma.

Anti-GD2 immunotherapy using mAb 3F8 has been shown to exert a small anti-tumor effect in chemoresistant neuroblastoma. The addition of a barley $\beta 1,3$-glucan to $3 \mathrm{~F} 8$ therapy in one study did not lead to increased CR3 mediated cytotoxicity (Modak et al., 2013). However, some patients developed anti-mouse antibody reaction. This was previously unobserved in
3F8 administration and associated with increased survival. Further investigation of the immunological effects of the combination is merited.

$\beta$-glucan binding of neutrophil CRs enhances specific humoral response targeting of tumor cells opsonised with complement iC3b and antibodies (Li et al., 2006; Novak and Vetvicka, 2009), constituting a mechanism of action for the synergy of mAbs and $\beta$-glucans in tumor shrinkage and enhanced host survival (Gelderman et al., 2004; Hong et al., 2004; Liu et al., 2009; Yan et al., 2005). Monoclonal antibodies with no inherent tumor regression activity may mediate regression which can be complete (Hong et al., 2004). For example, Herceptin and Erbitux are mAbs against Her2 and Her1 growth factors respectively which do not stimulate murine tumor regression unless administered with $\beta 1,3$ glucans (Hong et al., 2004). Rituxan performs similarly (Hong et al., 2004). Vaccines which elicit anti-tumor antibodies may have increased efficacy if $\beta 1,3$ glucan co-administration occurs (Hong et al., 2004; Liu et al., 2009; Yan et al., 2005), representing an area for future investigation.

\subsection{Vitamin $\mathrm{C}$}

Vitamin $C$ and $\beta 1,3$-glucans increase some of the same classes of immune responses: NK cell and macrophage activity amplification, specific antibody formation and cytokine levels (Schwager and Schulz, 1998; Vetvicka and Vetvickova, 2012a). When combined, vitamin $C$ and $\beta 1,3$-glucans produce synergistic increases in adaptive biological and immunological measures (Ditteova et al., 2003; Verlhac et al., 1998), most notably when the glucan is in liposomised form.

\subsection{Transresveratrol Trihydroxystilbene)}

(Trans-3,4',5-

Transresveratrol is a nonflavonoid polyphenol with independent cancer chemopreventiveimmunomodulatory properties, typically isolated from grape skin or Japanese knotweed (Vetvicka et al., 2007b). It has been found to ameliorate the side effects of administration of doxorubicin and cisplatin chemotherapy (Do Amaral et al., 2007; Vetvicka and Vetvickova, 2012a). Vetvicka et al. (2007b) combined transresveratrol with WGP and found synergistic potentiation of phagocytosis, proliferation of $\mathrm{CD}^{+} \mathrm{T}$ lymphocytes, production of potentially lymphocyte stimulating cytokines and splenic recovery from administration of 5-flourouracil. Synergistic upregulation of genes encoding for anti-apoptosis, cell growth and adhesion pathways (such as $\mathrm{Cdc} 42$ and NF-кB2) occurred. 


\subsection{Vitamin C Plus Transresveratrol}

A recent study used a combination of vitamin $\mathrm{C}$, transresveratrol and Glucan\#300 against murine lung cancer and breast cancer in order to evaluate the treatment potential of the combination (Vetvicka and Vetvickova, 2012a). The triple combination was found to be the strongest enhancer of granulocyte numbers, phagocytosis and antibody formation, achieving the greatest reduction in metastasis $(86 \%)$.

In a subsequent study, mercury or perfluorinated hydrocarbon poisoning were used to depress a range of immunological parameters in mice, including phagocytosis, NK cell activity, mitogen-induced proliferation and expression of $\mathrm{CD}$ markers, antibody formation and secretion of cytokines IL-6, IL-12 and IFN- $\gamma$ (Vetvicka and Vetvickova, 2012b). A vitamin $\mathrm{C} /$ transresveratrol/Glucan \#300 combination was shown to ameliorate immunosuppression.

In an additional study the same researchers compared five commercially available preparations, most of which combined $\beta 1,3$-glucans with other immunomodulators (Table 3), with the commercially available vitamin $\mathrm{C} /$ transresveratrol/Glucan \#300 combination "RVB $300 \AA$," in terms of levels of phagocytosis, respiratory burst (nitrite and superoxide production), IL-2 production, antibody response to ovalbumin and NK cell cytotoxicity (Vetvicka and Vetvickova, 2012c).

Only RVB 300® showed immunological activity across measures. Except for nitrite and superoxide anion formation, the other samples showed no such activity, despite their inclusion of $\beta 1,3$-glucans with some evidence base. This may be due to low doses of active, poor isolation techniques or component immunomodulators negating each other's efficacy.

\subsection{Humic Acids}

Humic acids are high molecular weight aromatic polymers found mainly in decomposing peat, some of which have anti-viral and lymphocyte-proliferative effects (Vetvicka et al., 2010). Vetvicka et al. (2010) used Glucan $\# 300 \circledR$ and $\beta 1,3$-glucan laminarin in combination with humic acids in testing. Two types of humic acids independently demonstrated synergy when combined in specific ratios with the $\beta$-glucans, in terms of production of specific cytokines (IL-2, IL-4, IL-5, IL-6 and TNF- $\alpha$ ) and of MPC-1 and phagocytosis functions of neutrophils and macrophages. The most strongly aromatic humic acid in combination with glucan achieved the greatest reduction of mouse breast cancer tumor weight in all tested samples: 92\% (Vetvicka et al., 2010).

Table 3. Products containing combined immunomodulators

\begin{tabular}{|c|c|}
\hline Product & Immunomodulatory components \\
\hline \multirow[t]{5}{*}{ Qivana® } & Reishi mushroom (Ganoderma lucidum) \\
\hline & Cordyceps mushroom (Cordyceps sinensis) \\
\hline & Coriolus mushroom [Krestin] (Coriolus versicolor) \\
\hline & Maitake mushroom (Grifola frondosa) \\
\hline & Zhu Ling mushroom (Polyporus umbellatus) \\
\hline BioBran $®$ & MGN-3 arabinoxylan compound from partially hydrolysed rice bran \\
\hline \multirow[t]{12}{*}{ Transfer factor Plus $®$} & Zinc (as zinc methione) \\
\hline & Transfer factor E-XF ${ }^{\mathrm{TM}}$ (extract of egg yolk and cow colostrum) \\
\hline & Nano Factor ${ }^{\mathrm{TM}}$ (nano-filtered cow colostrum) \\
\hline & Inositol hexaphosphate \\
\hline & B-Sitosterol and other polysterols \\
\hline & Aloe leaf gel extract \\
\hline & Olive leaf extract \\
\hline & Oat seed extract \\
\hline & Baker's yeast extract \\
\hline & Cordyceps sinensis mushroom extract \\
\hline & Maitake (Grifola frondosa) mushroom extract \\
\hline & Shiitake (Lentinus edodes) extract \\
\hline \multirow[t]{2}{*}{ Manapol Plus® } & Aloe gel powder \\
\hline & $\beta$-glucan from Maitake mushroom (Grifola frondosa) \\
\hline \multirow[t]{4}{*}{ Immunizen $®$} & Colostrum \\
\hline & Arabinogalactan \\
\hline & Lactoferrin \\
\hline & $\beta 1,3$ glucan from baker's yeast \\
\hline \multirow[t]{3}{*}{ RVB $300 ®$} & Glucan \#300 (Saccharomyces cerevisiae) \\
\hline & Resveratrol, \\
\hline & Vitamin $\mathrm{C}$ \\
\hline
\end{tabular}




\subsection{Ashwagandha (Withania Somnifera)}

Ashwagandha is an herbal extract used for stress reduction in traditional Indian medicine. When administered with Yukiguni Maitake extract (MD fraction) a synergistic inhibition of stress-related corticosterone increase and cytokine reduction occurred (Vetvicka and Vetvickova, 2011b), with implications for maintenance of a healthy immune function during stress related to a cancer diagnosis for a human patient.

\subsection{Whole Maitake (Grifola Frondosa) Powder}

In a previously referred to non-randomised clinical trial (Kodama et al., 2002a) administered Yukiguni Maitake MD Fraction® plus Yukiguni whole Maitake powder to liver, lung and breast cancer patients. They found $58.3-68.8 \%$ cancer regression or rigorously defined symptomatic improvement. Nanba (1997) had previously reported similar findings. In each study the combination of products achieved superior results to those produced with MD Fraction alone, although it is unclear as to whether this may be due to $\beta$-glucan dose increase due to addition of glucan in the whole powder, or synergy of MD fraction with a component or components of the whole powder.

\section{DISCUSSION}

Studies concerned with $\beta 1,3$-glucan product efficacies and synergies are usually confounded by the issues of unknown optimal dose and unknown degree of product standardisation. Although (Deng et al., 2009) established the human oral dose response curves for Maitake Gold 404® across a wide range of immunological parameters, in general it is unknown as to whether murine and human studies used optimal doses of the range of $\beta 1,3$-glucans tested, (and whether the optimal dose for one $\beta 1,3$ glucan is the same as that for another). Nevertheless, $\beta 1,3$-glucan products found to be efficacious as tumoricidal agents or carcinogenesis immunological mechanism inhibitors consistently include Betamune ${ }^{\circledR}$ (Vetvicka et al., 2008), Glucan \#300® and Maitake Gold 404® (Vetvicka and Vetvickova, 2005; Vetvicka and Vetvickova, 2007a; 2007b; Vetvicka and Vetvickova, 2009; Vetvicka and Vetvickova, 2010).

Maitake Gold 404® products are dilutions of Yukiguni Maitake MD fraction ${ }^{\circledR}$ and are produced by a range of US manufacturers (iHerb, 2014). Administration of a Maitake Gold 404® product in one study resulted in between sample variation in neutrophil stimulation and anomalous low IL-2 stimulation (Vetvicka and Vetvickova, 2005), suggesting product standardisation problems. Many additional marketed products contain non-patented substances claimed to be $\mathrm{MD}$ or $\mathrm{D}$ fraction, hence unknown doses of intact or authentic molecules of active (iHerb, 2014). Yukiguni Maitake MD Fraction $₫$ is extracted from bio farmed high $\beta 1,3$-glucan content specimens, under uniform optimal growing conditions, with rigorous quality control, resulting in standardisation (Deng et al., 2009; Kodama et al., 2002a). Glucan extraction involves fractionation so as to obtain a relatively pure product, which is patented and proprietary (Nanba and Kubo, 1997). So that studies use a known dose of effective MD fraction, it is recommended that only MD fraction obtaineddirectly from Yukiguni be used in future trials, rather than manufacturer diluted or look-alike products. To date, apart from one known study (Vetvicka and Vetvickova, 2005), only Japanese studies have used the original product, generally with very efficacious results (Inoue et al., 2002; Kodama et al., 2002a; 2002b; Masuda et al., 2012; Miyake et al., 2005). In addition, it is contrary to patient interests that numerous diluted products on the US market contain low levels of patented active compared with the original product and that recommended doses are subtherapeutic (Deng et al., 2009; iHerb, 2014).

Systematic identification of optimal dose for each $\beta 1,3$-glucan product must be determined (preferably in human subjects), before meaningful comparisons of product efficacies may be made. After optimal doses are identified, it is suggested that Yukiguni MD fraction ${ }^{\circledR}$, Glucan $\# 300 \AA$ and Betamune ${ }^{\circledR}$ effects be trialled across all immunological measures and tumor weight, so that conclusions on comparative immunological and anticancer effects may be derived.

Agents containing $\beta 1,3$-glucans, have shown synergy with conventional cancer treatments and produced amelioration of their adverse effects (Gu et al., 2005; Kodama et al., 2002a; Lin et al., 2004; 2010; 2009; Louie et al., 2010; Masuda et al., 2009; 2010; Nanba, 1997; Novak and Vetvicka, 2008; Sener et al., 2006; Vetvicka et al., 2007a). Although combinations of mAbs and $\beta 1,3$-glucans have been shown to produce synergistic tumoricidalactivity (Hong et al., 2004; Liu et al., 2009; Modak et al., 2013; Salvador et al., 2008; Yan et al., 2005), further research is needed to elucidate the specific cancer cell lines for which particular mAb- $\beta 1,3$-glucan combinations possess efficacy.

RVB300® ( $\beta$-glucan/transresveratrol/vitamin C) is a $\beta$-glucan-basedcombination immunomodulator product 
tested which demonstrated favourable immunological effects (Vetvicka and Vetvickova, 2012c). Maitake QOL Extract ${ }^{\circledR}$ is composed of Yukiguni whole Maitake powder and Yukiguni Maitake MD Fraction ${ }^{\circledR}$, combined in approximately the ratio shown by Nanbato produce cancer regression in human patients above that produced by MD Fraction alone (Kodama et al., 2002a). After the accurate determination of the MD fraction within the whole Maitake in QOL $囚$, it is recommended that Maitake QOL Extract ${ }^{\circledR}$ and RVB300® be comparatively trialled at optimal dose across immunological measures and tumor weight. Systematic elucidation of synergies between $\beta 1,3$-glucans and other immunomodulator combinationsis a promising area in product development.

\section{CONCLUSION}

Accumulated evidence shows that products containing $\beta 1,3$-glucans can be anti-carcinogenic immunomodulators which possess utility as adjunctive therapy with chemotherapy, other cancer therapies and other immunomodulators. Unfortunately, many studies suffer from experimental design issues including nonrandomisation and incomplete control of dose of specific glucan. It is recommended that these issues be addressed in further research.

\section{ACKNOWLEDGEMENT}

The researchers wishes to thank the following advisorwithout whom the writing of this study would not have been possible: Mr Bruce Muir, Managing Director, QOL Nutraceuticals Incorporated.

\section{REFERENCES}

ACS, 2014. Facts and figures. American Cancer Society. CMEC, 2006. Extracted ratified minutes. CMEC.

Cui, F.J., W.Y. Tao, Z.H. Xu, W.J. Guo and H.Y. Xu et al., 2007. Structural analysis of anti-tumor heteropolysaccharide GFPS1b from the cultured mycelia of Grifola frondosa GF9801. Bioresource Technol., 98: 395-401. DOI: 10.1016/j.biortech.2005.12.015

Deng, G., H. Lin, A. Seidman, M. Fornier and G. D'Andrea et al., 2009. A phase I/II trial of a polysaccharide extract from Grifola frondosa (Maitake mushroom) in breast cancer patients: Immunological effects. J. Canc. Res. Clin. Oncol., 135: 1215-1221. DOI: 10.1007/s00432-009-0562-Z
Ditteova, G., S. Velebny and G. Hrckova, 2003. Modulation of liver fibrosis and pathophysiological changes in mice infected with Mesocestoides corti (M. vogae) after administration of glucan and liposomized glucan in combination with vitamin C. J. Helminthol., 77: 219-226. DOI: 10.1079/JOH2002161

Do Amaral, C.L., H.D. Francescato, T.M. Coimbra, R.S. Costa and J.D. Darin et al., 2007. Resveratrol attenuates cisplatin-induced nephrotoxicity in rats. Arch. Toxicol., 82: 363-370. DOI: 10.1007/s00204007-0262-X

Gelderman, K.A., S. Tomlinson, G.D. Ross and A. Gorter, 2004. Complement function in mAbmediated cancer immunotherapy. Trends Immunol., 25: 158-164. DOI: 10.1016/j.it.2004.01.008

Gu, Y.H., Y. Takagi, T. Nakamura, T. Hasegawa and I. Suzuki et al., 2005. Enhancement of radioprotection and anti-tumor immunity by yeast-derived $\beta$-glucan in mice. J. Med. Food, 8: 154-158. DOI: 10.1089/jmf.2005.8.154

Hamano, K., H. Gohra, T. Katoh, Y. Fujimura and N. Zempo et al., 1999. The preoperative administration of lentinan ameliorated the impairment of natural killer activity after cardiopulmonary bypass. Int. J. Immunopharmacol., 21: 531-540. DOI: 10.1016/S0192-0561(99)00033-8

Hishida, I., H. Nanba and H. Kuroda, 1988. Antitumor activity exhibited by orally administered extract from fruit body of Grifola frondosa (maitake). Chem. Pharm. Bull., 36: 1819-1827. PMID: 3203420

Hong, F., J. Yan, J.T. Baran, D.J. Allendorf and R.D. Hansen et al., 2004. Mechanism by which orally administered $\beta$-1,3-glucans enhance the tumoricidal activity of antitumor monoclonal antibodies in murine tumor models. J. Immunol., 173: 797-806. DOI: 10.4049/jimmunol.173.2.797

iHerb, 2014 Search Results for "maitake d fraction". iHerb.com.

Inoue, A., N. Kodama and H. Nanba, 2002. Effect of maitake (Grifola frondosa) D-fraction on the control of the T lymph node Th-1/Th-2 proportion. Biol. Pharm. Bull., 25: 536-540. PMID: 11995941

Ito, K., Y. Masuda, Y. Yamasaki, Y. Yokota and H. Nanba, 2009. Maitake beta-glucan enhances granulopoiesis and mobilization of granulocytes by increasing G-CSF production and modulating CXCR4/SDF-1 expression. Int. Immunopharmacol., 9: 1189-1196. DOI: 10.1016/j.intimp.2009.06.007 
Jamois, F., V. Ferrières, J.P. Guégan, J.C. Yvin and D. Plusquellec et al., 2005. Glucan-like synthetic oligosaccharides: Iterative synthesis of linear oligo$\beta$-(1,3)-glucans and immunostimulatory effects. Glycobiology, 15: 393-407. DOI: 10.1093/glycob/cwi020

Kawagishi, H., R. Inagaki, T. Kanao and T. Mizuno, 1989. Fractionation and antitumor activity of the water-in-soluble residue of Agaricusblazei fruiting bodies. Carbohydrate Res., 186: 267-273. DOI: 10.1016/0008-6215(89)84040-6

Kodama, N., K. Komuta and H. Nanba, 2002a. Can Maitake MD-fraction aid cancer patients? Alt. Med. Rev., 7: 236-239. PMID: 12126464

Kodama, N., K. Komuta, N. Sakai and H. Nanba, 2002b. Effects of D-Fraction, a polysaccharide from Grifola frondosa on tumor growth involve activation of NK cells. Biol. Pharm. Bull., 25: 1647-1650. DOI: 10.1248/bpb.25.1647

Li, B., D. Allendorf, R. Hansen, J. Marroquin and C. Ding et al., 2006. Yeast beta-Glglucan amplifies phagocyte killing of iC3b-opsonized tumor cells via complement receptor 3-Syk-phosphatidylinositol 3kinase pathway. J. Immunol., 177: 1661-1669. DOI: 10.4049/jimmunol.177.3.1661

Lin, H., E. De Stanchina, X.K. Zhou, F. Hong and A. Seidman et al., 2010. Maitake beta-glucan promotes recovery of leukocytes and myeloid cell function in peripheral blood from paclitaxel hematotoxicity. Canc. Immunol. Immunotherapy, 59: 885-897. DOI: 10.1007/s00262-009-0815-3

Lin, H., E. De Stanchina, X.K. Zhou, Y.H. She and D. Hoang et al., 2009. Maitake betaglucan enhances umbilical cord blood stem cell transplantation in the NOD/SCID mouse. Exp. Biol. Med., 234: 342-353. DOI: $10.3181 / 0807-R M-226$

Lin, H., S.W. Cheung, M. Nesin, B.R. Cassileth and S. Cunningham-Rundles, 2007. Enhancement of umbilical cord blood cell hematopoiesis by maitake beta-glucan is mediated by granulocyte colonystimulating factor production. Clin. Vaccine Immunol., 14: 21-27. DOI: 10.1128/CVI.00284-06

Lin, H., Y.Y. She, B.R. Cassileth, F. Sirotnak and S.C. Rundles, 2004. Maitake beta-glucan MD-fraction enhances bone marrow colony formation and reduces doxorubicin toxicity in vitro. Int. Immunopharmacol., $\quad 4$ : 91-99. DOI: 10.1016/j.intimp.2003.10.012
Liu, J., L. Gunn, R. Hansen and J. Yan, 2009. Yeastderived $\beta$-glucan in combination with anti-tumor monoclonal antibody therapy in cancer. Recent Patents Anti-Cancer Drug Discovery, 4: 101-109. DOI: $10.2174 / 157489209788452858$

Louie, B., S. Rajamahanty, J. Won, M. Choudhury and S. Konno, 2010. Synergistic potentiation of interferon activity with maitake mushroom dfraction on bladder cancer cells. Br. J. Urol. Int., 105: 1011-1015. DOI: 10.1111/j.1464410X.2009.08870.x

Lowman, D., D.A. Ferguson and D.L. Williams, 2003. Structural characterization of (1-3)- $\beta$-D-glucans isolated from blastospore and hyphal forms of Candida albicans. Carbohydrate Res., 338: 14981496. DOI: 10.1016/S0008-6215(03)00169-1

Masuda, Y., K. Ito, M. Konishi and H. Nanba, 2010. A polysaccharide extracted from Grifola frondosa enhances the anti-tumor activity of bone marrowderived dendritic cell-based immunotherapy against murine colon cancer. Canc. Immunol. Immunotherapy, 59: 1531-1541. DOI: 10.1007/s00262-010-0880-7

Masuda, Y., M. Inoue, A. Miyata, S. Mizuno and H. Nanba H, 2009. Maitake $\beta$-glucan enhances therapeutic effect and reduces myelosupression and nephrotoxicity of cisplatin in mice. Int. Immunopharmacol., 9: 620-626. DOI: 10.1016/j.intimp.2009.02.005

Masuda, Y., T. Togo, S. Mizuno, M. Konishi and H. Nanba, 2012. Soluble $\beta$-glucan from Grifola frondosa induces proliferation and Dectin1/Syksignaling in resident macrophages via the GMCSF autocrine pathway. J. Leuk. Biol., 91: 547-556. DOI: $10.1189 /$ jlb.0711386

Masuda, Y., Y. Murata, M. Hayashi and H. Nanba, 2008. Inhibitory effect of MD-Fraction on tumor metastasis: Involvement of NK cell activation and suppression of Intercellular Adhesion Molecule (ICAM)-1 expression in lung vascular endothelial cells. Biol. Pharm. Bull., 31: 1104-1108. PMID: 18520039

Miyake, N., Y. Masuda, Y. Murata, M. Inoue and K. Matuda et al., 2005. Low molecular weight maitake MD-Fraction (Klasma-MD) hydrolyzed with endob-1,6-glucanase of Trichodermaharzianum induces antitumor activities. Mycoscience, 47: 78-83. DOI: 10.1007/s10267-005-0274-4 
Modak, S., B.H. Kushner, K. Kramer, A. Vickers and I.Y. Cheung et al., 2013. Anti-GD2 antibody 3F8 and barley-derived $(1 \rightarrow 3),(1 \rightarrow 4)-\beta$-D-glucan. A Phase I study in patients with chemoresistantneuroblastoma. Oncoimmunology, 2: e23402-1-e23403-8. DOI: 10.4161/onci.23402

Muir, B., 2012. Maitake QOL Extract. ${ }^{\text {TM }}$

Nanba, H. and K. Kubo, 1997. Antitumor substance extracted from Grifola. PatentGenius.

Nanba, H., 1997. Maitake D-fraction: Healing and preventive potential for cancer. J. Orthomol. Med., 12: 43-50.

Nanba, H., A. Hamaguchi and H. Kuroda, 1987. The chemical structure of an antitumor polysaccharide in fruit bodies of Grifola frondosa (maitake). Chem. Pharm. Bull., 35: 1162-1168. PMID: 3607939

Novak, M. and V. Vetvicka, 2008. $\beta$-glucans, history and the present: Immunomodulatory aspects and mechanisms of action. J. Immunotoxicol., 5: 47-57. DOI: $10.1080 / 15476910802019045$

Novak, M. and V. Vetvicka, 2009. Glucans as biological response modifiers. Endocr. Metab. Immune Disord. Drug Targets, 9: 67-75. PMID: 19275682

Ohno, N., Y. Adachi, I. Suzuki, K. Sato and S. Oikawa et al., 1986. Characterization of the antitumor glucan obtained from liquid-cultured Grifola frondosa. Chem. Pharm. Bull., 34: 17091715. PMID: 3719872

Otake, Y., S. Soundararajan, T.K. Segupta, E.A. Kio and J.C. Smith et al., 2007. Overexpression of nucleolin in chronic lymphocytic leukemia cells induces stabilization of bcl2 mRNA. Blood, 109: 3069-3075. DOI: 10.1182/blood-2006-08-043257

Salvador, C., B. Li, R. Hansen, D.E. Cramer and M. Kong et al., 2008. Yeast-derived $\beta$-glucan augments the therapeutic efficacy mediated by anti-vascular endothelial growth factor monoclonal antibody in human carcinoma xenograft models. Clin. Canc. Res., 14: 1239-1247. DOI: 10.1158/10780432.CCR-07-1169

Schwager, J. and J. Schulz, 1998. Modulation of interleukin production by ascorbic acid. Vet. Immunol. Immunopathol., 64: 45-57. DOI: 10.1016/S0165-2427(98)00120-2

Sener, G., E. Eksioglu-Demiraop, M. Cetiner, F. Ercan and B.C. Yegen, 2006. Beta-glucan ameliorates methotrexate-induced oxidative organ injury via its antioxidant and immunomodulatory effects. Eur. J. Pharmacol., 542: 170-178. DOI: 10.1016/j.ejphar.2006.02.056
Shomori, K., M. Yamamoto, I. Arifuku, K. Teramachi and H. Ito, 2009. Antitumor effects of a watersoluble extract from maitake (Grifola frondosa) on human gastric cancer cell lines. Oncol. Rep., 22: 615-620. DOI: 10.3892/or_00000480

Tada, R., T. Harada, N. Nagi-Miura, Y. Adachi and M. Nakajima et al., 2007. NMR characterization of the structure of a $\beta$ - $(1 \rightarrow 3)$-d-glucan isolate from cultured fruit bodies of Sparassiscrispa. Carbohydrate Res., 342: 2611-2618. DOI: 10.1016/j.carres.2007.08.016

Tada, R., Y. Adachi, K. Ishibashi and N. Ohno, 2009. An unambiguous structural elucidation of a 1,3-b-Dglucan obtained from liquid-cultured Grifola frondosa by solution NMR experiments. Carbohydrate Res., 344: 400-404. DOI: 10.1016/j.carres.2008.11.005

Verlhac, V., A. Obach, J. Gaubaudan, W. Schüep and R. Hole, 1998. Immunomodulation by dietary vitamin $\mathrm{C}$ and glucan in rainbow trout (Oncorhynchusmykiss). Fish Shellfish Immunol., 8: 409-424. DOI: 1050-4648/98/060409+16

Vetvicka, V. and J.C. Yvin, 2004. Effects of marine $\beta$ 1,3 glucan on immune reactions. Int. Immunopharmacol., 4: 721-730. DOI: 10.1016/j.intimp.2004.02.007

Vetvicka, V. and J. Vetvickova, 2005. Immunostimulating properties of two different $\beta$ glucans isolated from maitake mushrooms (Grifola frondosa). J. Am. Nutraceut. Assoc., 8: 33-39.

Vetvicka, V. and J. Vetvickova, 2007a. Physiological effects of different types of $\beta$-glucan. Biomed. Pap., 151: 225-231. PMID: 18345255

Vetvicka, V. and J. Vetvickova, 2007b. An evaluation of the immunological activities of commercially available $\beta 1,3$ Glucans. J. Am. Nutraceut. Assoc., 10: 25-31.

Vetvicka, V. and J. Vetvickova, 2009.Effects of glucan on immunosuppressive actions of mercury. J. Med. Food, 12: 1098-1104. DOI: 10.1089/jmf.2008.0273

Vetvicka, V. and J. Vetvickova, 2010. $\beta 1,3$-glucan: Silver bullet or hot air? Open Glycosci., 3: 1-6.

Vetvicka, V. and J. Vetvickova, 2011a. $\beta(1-3)$-D-glucan affects adipogenesis, wound healing and inflammation. Oriental Pharm. Exp. Med., 11: 169175. DOI: 10.1007/s13596-011-0024-4

Vetvicka, V. and J. Vetvickova, 2011b. Immune enhancing effects of WB365, a novel combination of Ashwagandha (Withania somnifera) and Maitake (Grifola frondosa) extracts. North Am. J. Med. Sci., 3: 320-324. DOI: 10.4297/najms.2001.3320 
Vetvicka, V. and J. Vetvickova, 2012a. Combination of glucan, resveratrol and vitamin $\mathrm{C}$ demonstrates strong anti-tumor potential. Anticancer Res., 32: 8188. PMID: 22213291

Vetvicka, V. and J. Vetvickova, 2012b. Glucanresveratrol-vitamin $\mathrm{C}$ combination offers protection against toxic agents. Toxins, 4: 1301-1308. DOI: 10.3390/toxins 4111301

Vetvicka, V. and J. Vetvickova, 2012c. Comparison of immunological properties of various bioactive combinations. Biomed. Papers, 156: 218-223. PMID: 23069887

Vetvicka, V., A. Vashishta, S. Saraswat-Ohri and J. Vetvickova, 2008. Immunological effects of yeast and mushroom derived $\beta$-glucans. J. Med. Food, 11: 615-622. DOI: 10.1089/jmf.2007.0588

Vetvicka, V., K. Terayama, R. Mandeville, P. Brousseau and B. Kournikakis et al., 2002. Pilot study: Orally administered yeast $\beta 1,3$-glucan prophylactically protects against anthrax infection and cancer in mice. J. Am. Nutraceut. Assoc., 5: 1-5.

Vetvicka, V., B. Dvorak, J. Vetvickova, J. Richter and J. Krizan et al., 2007a. Orally-administered marine $(1 \rightarrow 3)-\beta$-D-glucan Phycarine stimulates both humoral and cellular immunity. Int. J. Biol. Macromol., 40: 291-298. DOI: 10.1016/j.ijbiomac.2006.08.009

Vetvicka, V., T. Volny, S. Saraswat-Ohri, A. Vashishta and Z. Vancikova at al., 2007b. Glucan and resveratrol complex-possible synergistic effects on immune system. Biomed. Pap., 151: 41-46. PMID: 17690738
Vetvicka, V., R. Baigorri, A.M. Zamarreño, J.M. GarciaMina and J.C. Yvin, 2010. Glucan and humic acid: Synergistic effects on the immune system. J. Med. Food, 13: 863-869. DOI: 10.1089/jmf.2009.0178

Vetvicka, V., J. Richter, V. Svozil, L.R. Dobiášová and V. Král, 2013. Placebo-driven clinical trials of yeast-derived $\beta$ - $(1,3)$ glucan in children with chronic respiratory problems. Ann. Translat. Med., 1: 26-26. DOI: 10.3978/j.issn.2305-5839.2013.07.01

Wu, M.J., T.L. Cheng, S.Y. Cheng, T.W. Lian and L. Wang et al., 2006. Immunomodulatory properties of Grifola frondosa in Submerged Culture. J. Agric. Food Chem., 54: 2906-2914. DOI: 10.1021/jf052893q

Xu, H., J.H. Liu, Z.Y. Shen, Y. Fei and X.D. Chen, 2010. Analysis of chemical composition, structure of Grifola frondosa polysaccharides and its effect on skin TNF- $\alpha$ levels, $\operatorname{lgG}$ content, T lymphocytes rate and caspase-3 mRNA. Carbohydrate Pol., 82: 687691. DOI: 10.1016/j.carbpol.2010.05.035

Yan, J., D.J. Allendorf and B. Brandley, 2005. Yeast Whole Glucan Particle (WGP) beta-glucan in conjunction with antitumor monoclonal antibodies to treat cancer. Expert Op. Biol. Ther., 5: 691-702. DOI: 10.1517/14712598.5.5.691

Zhang, M., S.W. Cui, P.C.K. Cheung and Q. Wang, 2007. Antitumor polysaccharides from mushrooms: A review on their isolation process, structural characteristics and antitumor activity. Trends Food Sci. Tech., 18: 4-19. DOI: 10.1016/j.tifs.2006.07.013 